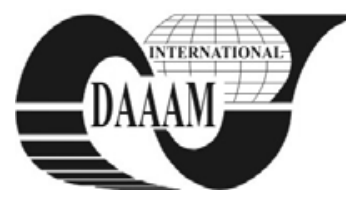

Annals of DAAAM for 2011 \& Proceedings of the 22nd International DAAAM Symposium, Volume 22, No. 1, ISSN $1726-9679$ ISBN 978-3-901509-83-4, Editor B. Katalinic, Published by DAAAM International, Vienna, Austria, EU, 2011 Make Harmony between Technology and Nature, and Your Mind will Fly Free as a Bird Annals \& Proceedings of DAAAM International 2011

\title{
CONFIRMATION OF MATHEMATICAL MODEL OF HELMHOLTZ COILS ON THE REAL CONSTRUCTIONS
}

\author{
UHER, M[iroslav]; FIALKA, J[iri] \& VAGNER, M[artin]
}

\begin{abstract}
This paper deals with distribution of magnetic field inside paired coils in Helmholtz arrangement. Helmholtz coils consist of two separate coils. Their mutually arrangements is advantageous for generation of uniform magnetic field. The simulation of field uniformity was realized. Further, the Helmholtz coils was made and the amplitude of magnetic field vector was measured. The comparison between simulation and really obtained results is discussed in this paper

Key words: Helmholtz coils, uniform magnetic field, COMSOL simulation, magnetic measurements
\end{abstract}

\section{INTRODUCTION}

The paired coils in Helmholtz arrangements are commonly used in laboratories for creating of uniform magnetic field. Other method for creating of homogenic magnetic field is e.g. spherical coils. The coils are named in honour of the german scientists Herman von Helmholtz, who dealed among others with electromagnetism. Helmholtz coils in one axis are consisted from two identical coils with defined radius $\mathrm{R}$. The centers of both coils are placed on the same axis in distance, which is equal to radius $\mathrm{R}$. The uniform magnetic field can be used for some magnetic measurements, including sensor calibration or measuring of earth magnetic field (Cacak and Craig, 1969), (Bronaugh, 1995).

For the exact calculation of magnetic induction value in any point inside Helmholtz coils it is necessary to use Bessel functions. The simpler evaluation of magnetic field can be made along the axis of coils. A derivation of relation for point, which is placed on coils axis in distance R/2 from both coils is very simple. The derivation of this relation begins with equation for computing of magnetic field $B$ inside single wire loop, which is derived from the Biot-Savart law (Trout, 1988):

$$
B=\frac{\mu_{0} I R^{2}}{2\left(R^{2}+x^{2}\right)^{\frac{3}{2}}}
$$

where $\mu_{0}$ is permeability of vacuum, $I$ is coil current, $R$ is coil radius and $\mathrm{x}$ is distance of investigated point on axis from one of coils. Each coil usually consist of more than one wire loop, that is the rason why we used in equations $n I$, where $n$ means number of loops. We can use also this substitution: the center of Helmholtz coils is placed in distance $x$, which is equal to $R / 2$. Because the Helmholtz coils is composition of two coils we have to multiply above mentioned equation by coefficient 2 . After above described substitution we can obtain following equation:

$$
B=\left(\frac{4}{5}\right)^{\frac{3}{2}} \frac{\mu_{0} n I}{R}
$$

The maint target of this paper is comparison between mathematical model created on base of numerical simulations and physical realisation of Helmholtz coils. We have focused this research on uniformity of magnetic field and the diferencies in determination of coils conversion constant.

\section{EXPERIMENTAL}

\subsection{Measurement in Helmholtz coils}

The suitable paired coils in Helmholtz arrangement was made for our laboratory in accordance with our proposition. The whole construction is made of electrically and magnetically non-conductive materials, that allow using of Helmholtz coils for AC applications. The skelet of both coils is made of hardened cotton fabric (textit) and the coils are fasted together by polyamide screwed bars. At the base of each coil there are terminals for wire connection. The radius $R$ of coil is equal to 0,2 m. Each coil contains 80 wire loops, which were made of enameled copper conductor. Maximally current rating of coils is approximately $10 \mathrm{~A}$. Connection diagram which was used for all measurement is shown on Figure 1.

Uniformity of magnetic field inside Helmholtz coils was measured during first part of our experiment. For measurements was used gaussmeter F.W.Bell model 5080. Magnetic field was investigated in 2D area $30 \times 30 \mathrm{~cm}$, which was situated symmetrically from center of Helmholtz coils. The distance of measuring points was in both axes $5 \mathrm{~cm}$. The measurement was proceeded for interval of supply currents from $1 \mathrm{~A}$ to $8 \mathrm{~A}$ with resolution $1 \mathrm{~A}$. The results of this part of the experiment were 3D graphs, which show distribution of magnetic field in Helmholtz coils. For example, the uniformity of magnetic field on the coils axis is shown in graph on Figure 2.

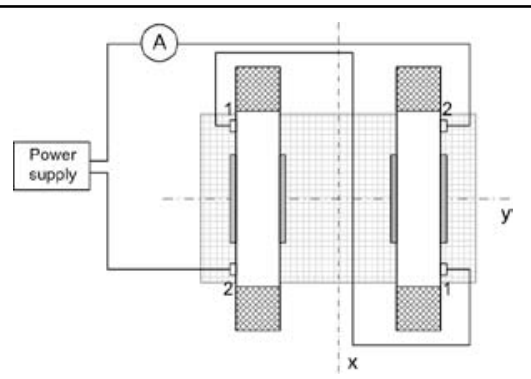

Fig. 1. Connection diagram of Helmholtz coils (Boll and Overshoott, 1989)

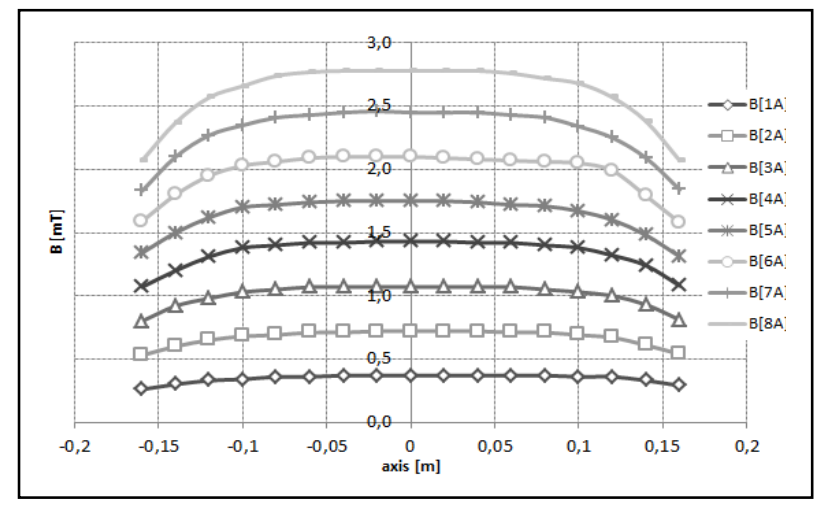

Fig. 2. Magnetic field B vs.position on axis, parametr is current 


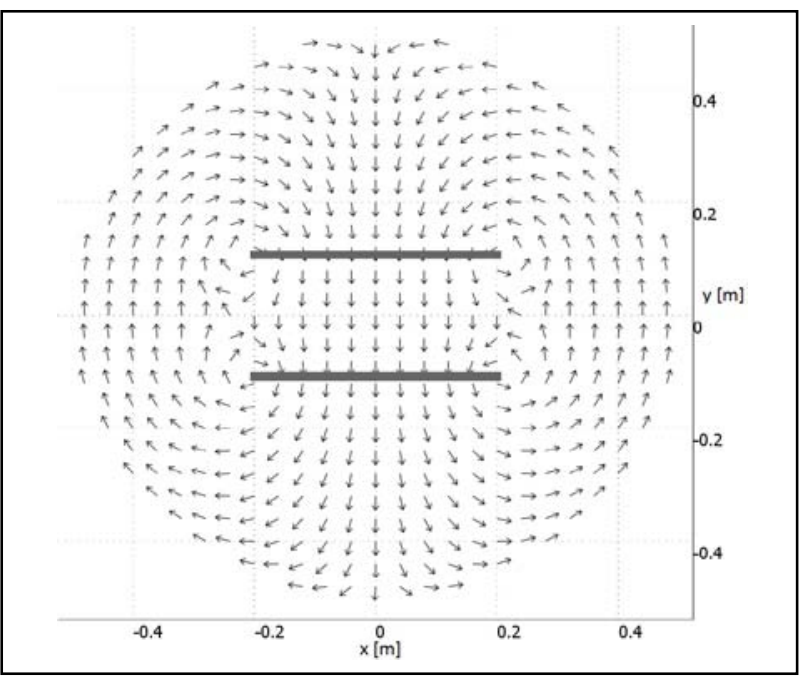

Fig. 3. The direction of magnetic field vectors from COMSOL simulation

\subsection{Magnetic field simulations}

For manufactured Helmholtz coils and their physical parameters were performed numerical simulations. These simulations were made using multiphysics simulation software COMSOL. With the aid of this simulation software were obtained distributions of magnetic field in coils for all measured points and currents. For example, the magnetic field lines diagram was created by the use of simulation. The diagram is shown in Figure 3. The direction of magnetic field vectors well corresponds with theoretically assumptions (Kaminishi and Nawata, 1981).

\subsection{Comparison of achieved results}

The measurement and the simulation were purposely designated for their mutually comparison. Comparison was made in the same points.

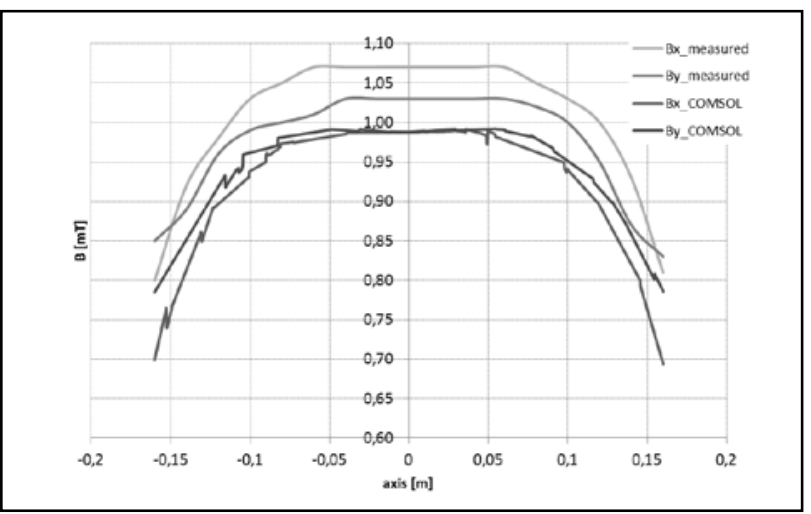

Fig. 4. The comparison of magnetic field B for two axes

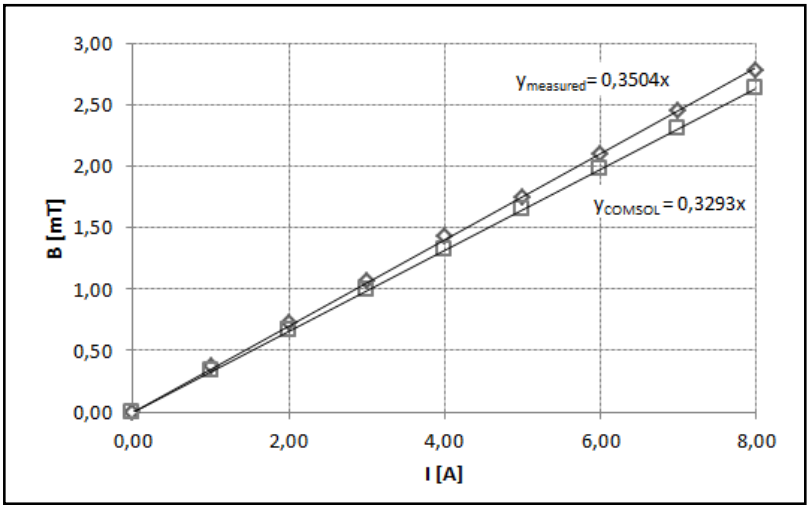

Fig. 5. The comparison of Helmholtz coils constant [mT/A]
Initially, the uniformity of magnetic field was comparised from 3D graphs. It was found out that magnetic field from simulation very well corresponds to results achieved from measurements. In both cases, the area in which could be magnetic field considered uniform has the same dimensions. The area has dimensions approximately $20 \times 10 \mathrm{~cm}$ for all currents and was located towards the coils axis by shorter side. The magnetic field was not uniform outside this area. There was an increase of field in proximity of coil, on the contrary in area far from center was observed decreasing of field.

Next, the comparison of dependencies of magnetic field $B$ on distance $x$ was accomplished. Each comparison (where the parameter was supply current) between measurements and simulation was made for both axes. For example, the comparison for current $3 \mathrm{~A}$ is shown on Figure 4. The matching for the others currents were similar. In general, the computed magnetic field was lower almost in all cases than the measured field.

Finally, the comparison of the Helmholtz coils conversion costant was made. This constant expressed relation between measured magnetic field and supply current. Its knowledge is important e.g. for Earth magnetic field measurement. The comparison of this constant determined from measurement and simulation is introduced in Figure 5. The results correspond with the results from previous analysis.

\section{CONCLUSION}

The partial uniformity of magnetic field for paired coils in Helmholtz arrangements was confirmed. The measurement and the simulation agree in achieved values of magnetic field. Therefore the Helmholtz coils could be used as a source of predicable uniform magnetic field in laboratory practises.

\section{ACKNOWLEDGEMENTS}

This paper was supported in part by the Grant Agency of the Czech Republic (102/09/H082 and 102/09/H081 SYNERGY - Sensors and Intelligent Sensor Systems) and by grant "Supporting Research of Modern Methods and Approaches in Automation" from the Internal Grant Agency of Brno University of Technology (grant No. FEKT-S-11-6).

\section{REFERENCES}

Boll, R., Overshoott, K. J., (1989). Sensors: A Comprehesive Survey, Magnetic Sensors, vol. 5, pg. 513, VCH Verlagsgesellschaft mbH. Germany, ISBN 3-527-26771-9

Bronaugh, E. L., (1995). Helmholtz coils for calibration of probes and sensors: limits of magnetic field accuracy and uniformity. 1995 IEEE International Symposium on Electromagnetic Compatibility, Atlanta - USA, pg. 72 - 76, ISBN: 0-7803-2573-7, DOI: 10.1109/ISEMC.1995.523521

Cacak, R. K., Craig, J. R., (1969). Magnetic Field Uniformity around Near Helmholtz Coil Configurations. Review of Scientific Instruments, vol. 40, issue 11, pg. $1468-1470$, November 1969. ISSN: 0034-6748, DOI: 10.1063/ 1.1683829

Kaminishi, K., Nawata, S., (1981). Practical method of improving the uniformity of magnetic fields generated by single and double Helmholtz coils. Review of Scientific Instruments, vol. 52, issue 3, pg. 447 - 453, Mar 1981. ISSN: 0034-6748, DOI: 10.1063/1.1136609

Trout, S. R., (1988). Use of Helmholtz coils for magnetic measurements. IEEE Transaction of Magnetics, vol. 24, issue 4, pg. 2108 - 2111, July 1988. ISSN: 0018-9464, DOI: $10.1109 / 20.3411$ 